\title{
Encoding multiple orientations in a recurrent network
}

\author{
Richard S. Zemel ${ }^{\mathrm{a}, *}$, Jonathan Pillow ${ }^{\mathrm{b}}$ \\ ${ }^{a}$ Department of Psychology, University of Arizona, Tucson, AZ 85721, USA \\ ${ }^{\mathrm{b}}$ Center for Neural Science, New York University, NY, USA
}

Accepted 13 January 2000

\begin{abstract}
Models containing recurrent connections amongst the cells within a population can account for a range of empirical data on orientation selectivity in striate cortex. However, existing recurrent models are unable to veridically encode more than one orientation at a time. Underlying this inability is an inherent limitation in the variety of activity profiles that can be stably maintained. We propose a new recurrent model that can form a broader range of stable population activity patterns. We demonstrate that these patterns preserve information about multiple orientations present in the population inputs. This preservation has significant computational consequences when information encoded in several populations must be integrated to perform behavioral tasks, such as visual discrimination. (C) 2000 Elsevier Science B.V. All rights reserved.
\end{abstract}

Keywords: Population codes; Orientation selectivity; Recurrent network models

\section{Introduction}

An important debate in computational neuroscience centers on the origin of selectivities in populations of cortical cells. A focus of this debate has been an extensive set of empirical data on orientation selectivity in primary visual cortex. A central question concerns how the observed sharp tuning of striate cells arises from the broadly tuned purely excitatory LGN input that grows monotonically with contrast. One class of network models posits that the sharp tuning is primarily due to the effects of recurrent connections within the striate population. These recurrent models can account for a wide range of the empirical data (reviewed in [5]).

\footnotetext{
* Corresponding author.

E-mail addresses: zemel@u.arizona.edu (R.S. Zemel), pillow@cns.nyu.edu (J. Pillow).
} 
Carandini and Ringach [1] studied a recurrent model - a simplified version of an earlier model proposed by Somers et al. [4] — that replicates many observed characteristics of a hypercolumn of striate cells. The core of their model is a single equation implementing a feedback filter with a center-surround weighting function. Their model successfully and succinctly captures a range of results, including sharp orientation tuning and contrast-invariant tuning width. However, when the input to the cell population contains two orientations, their model exhibits peculiar responses: if the two orientations differ by less than $45^{\circ}$, the model responds as if the input contained a single orientation at the mean of the two values; if the angular difference between the two orientations exceeds $45^{\circ}$, the model responds as if they were nearly orthogonal. The model also cannot signal the presence of three orientations, and creates spurious orthogonal orientations to noisy single orientation inputs. These authors analyzed their model and showed that these effects of attraction and repulsion between orientations are unavoidable in a broad class of recurrent models of orientation selectivity.

This model thus makes strong predictions about the visual response of cortical neurons to stimuli containing multiple orientations. Relevant empirical data are scarce. DeAngelis et al. [2] found that the response of a striate cell to its optimal orientation is reduced when the stimulus contains a second orientation. Cell responses to pairs of non-optimally oriented stimuli are not known. Nonetheless, the predictions of the model appear dubious: it predicts that the neurons will be unable to veridically encode multiple orientations, even when the orientations differ by nearly $45^{\circ}$. The aim of the model presented here is to explore if a broader range of activity patterns could be maintained in the population for multiple-orientation stimuli. We also studied the decoding of these patterns to determine whether information about the orientations is preserved.

\section{Methods}

The dynamics in our model are very similar to the Carandini-Ringach $(\mathrm{C}-\mathrm{R})$ model, with two modifications. Both models contain a group of cortical neurons identical except in preferred orientation $\left(-90^{\circ}, 90^{\circ}\right)$, where the response of neurons with preferred orientation $\phi$ to a stimulus containing orientation $\theta$ depends only on $(\theta-\phi)$. Their model is governed by the following equation:

$$
\tau \delta V / \delta t+V=V^{\mathrm{LGN}}+V^{\mathrm{EXC}}-V^{\mathrm{INH}}
$$

where $V(\theta, t)$ is the membrane potential of cells in orientation column $\theta$ at time $t, \tau$ is the membrane time constant, $V^{\mathrm{LGN}}$ is the excitatory input from LGN, and $V^{\mathrm{EXC}}$ and $V^{\mathrm{INH}}$ represent intra-cortical excitation and inhibition. These latter two quantities are obtained by convolving the responses of the cells with a narrowly tuned set of excitatory, and a broader profile of inhibitory weights, respectively. The combination of these two sets of weights produces a center-surround profile. Finally, each cell's response $R$ is a rectified linear function of its potential $V$. 


\section{Our model utilizes}

(1) optimized recurrent weights, both excitatory and inhibitory. The optimization procedure uses a set of examples, each consisting of the LGN input appropriate to a stimulus containing 1,2, or 3 bars (or gratings) at random orientations, and target output values for the model striate cells. The weights are modified such that the targets are the stable activity profiles of the differential state update equation (Eq. (1)). The target outputs are obtained by convolving each cell's tuning function, i.e., its expected response to a single-bar stimulus as a function of its orientation, with the set of orientations present in the stimulus. These targets are based on the assumption that a cell's response to a stimulus containing a pair of oriented bars is a scaled sum of its response to the individual bars; this assumption applies to MT cell responses to multiple-direction stimuli $[6,7]$.

(2) nonlinear activation function. We used the function proposed by Zhang [9]: $R=a \log (1+\exp (b(V+c)))^{d}$ (where $a, b, c$, and $d$ are constants) because it is easy to analytically invert, which facilitates the weight optimization procedure.

A third important addition to our model involves a different way of interpreting the activities of the striate cells. In order to determine what information about orientation is present in the model cell responses, we apply a statistical decoding method that can naturally handle multiple-valued inputs [8]. This method takes into account the cells' tuning functions in finding the set of inputs most likely to have generated the observed responses. A key feature of this method is that unimodal response profiles do not necessarily indicate that only one value is present in the input; instead, a broad unimodal profile is consistent with a stimulus containing two nearby orientations.

\section{Results}

The response of the model to stimuli containing two orientations, of varying angular difference, is shown in Fig. 1. Our model forms different stable activity patterns to all three pairs, and the decoding method accurately recovers the original orientations present in the stimulus. In contrast, the $\mathrm{C}-\mathrm{R}$ model is unable to veridically encode any of these stimuli.

Our model is also able to maintain a stable response profile for stimuli containing three orientations, as well as a clean unimodal response to noisy single-orientation stimuli (see Fig. 2). In both cases, the decoding method reconstructs the orientations in the stimulus.

The primary difference between the models is highlighted in Fig. 3, which represents their responses to all possible two-orientation stimuli. Each horizontal slice represents the response to a particular stimulus, and the angular spread between the two orientations increases from $0^{\circ}$ to $90^{\circ}$ along the $y$-axis. The $\mathrm{C}-\mathrm{R}$ model contains a discontinuity near $45^{\circ}$, as the population response jumps from uni- to bimodal, while our model contains a smooth transition from one to two orientations. 


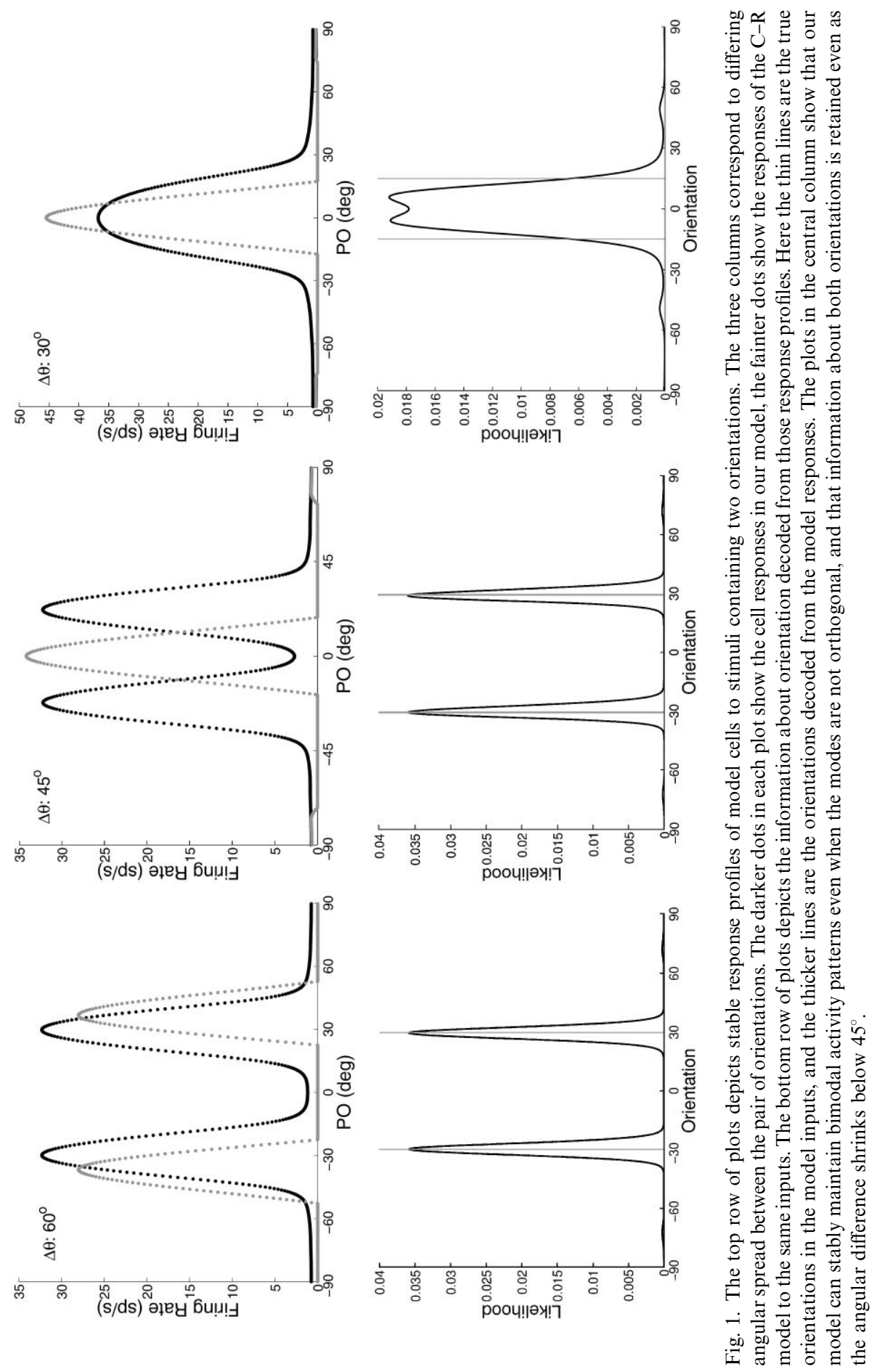



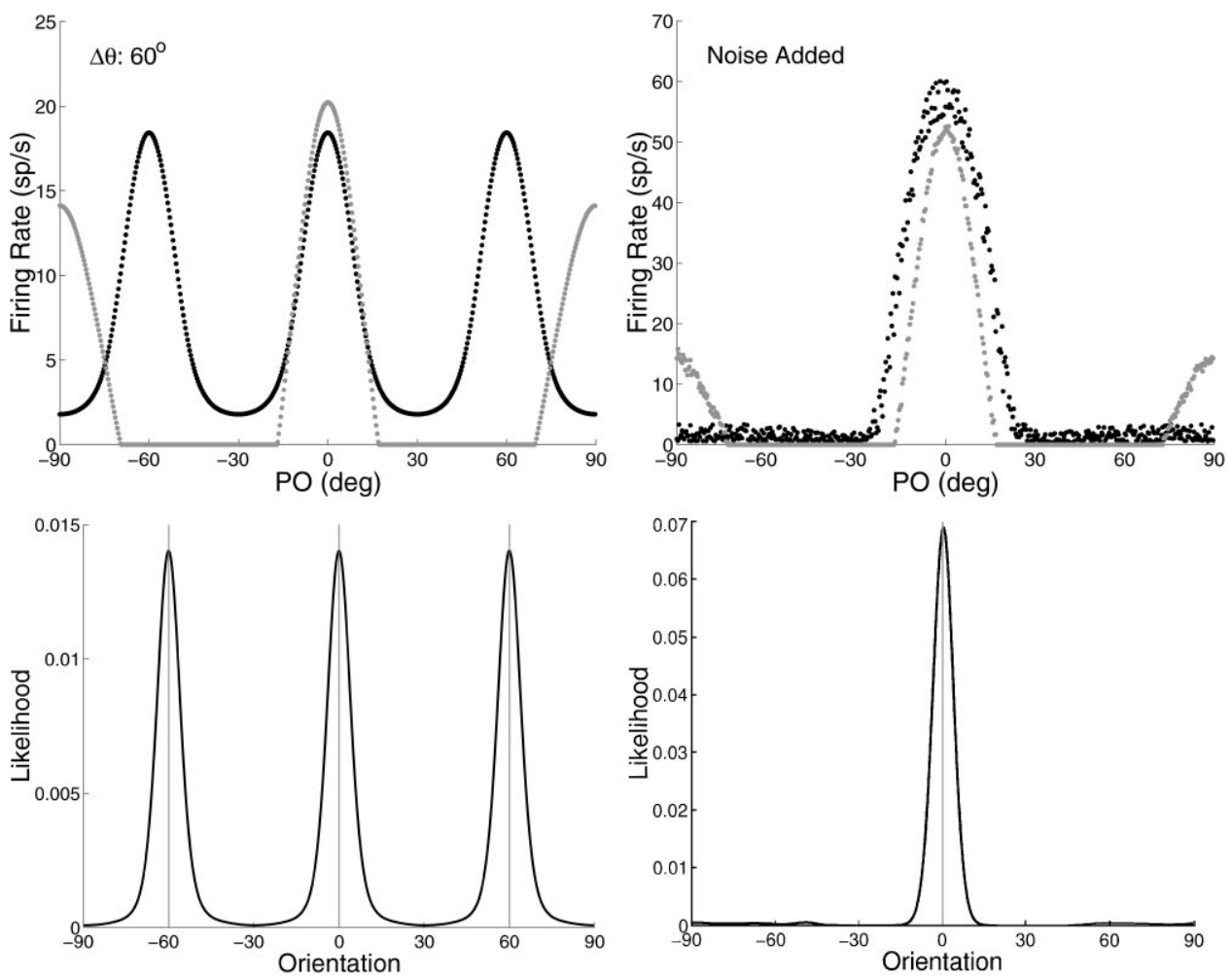

Fig. 2. The response of both models to inputs containing three orientations (lines labeled as in Fig. 1), and a single orientation plus noise is shown above, and the decoding of our model below.

The optimized recurrent weights in our model (see Fig. 4) have a similar shape to the center-surround weights used by most recurrent models. The wiggles in the surround of the weights gives them some power at higher frequencies. We are currently analyzing these differences in greater detail. A crucial difference not apparent in this figure is that the relative strength of the recurrent versus feedforward weights is smaller in our model than the C-R model. This difference likely underlies our model's reduced ability to remove noise and remain strongly contrast invariant, but enhanced ability to encode multiple orientations.

\section{Discussion}

We have proposed a novel model of orientation selectivity in striate cortex that can support a variety of response profiles, and veridically encodes multiple orientations. Our model can replicate the cross-orientation effects observed in one of the only empirical investigations into primary visual cortex responses to multiple orientations [2]. 

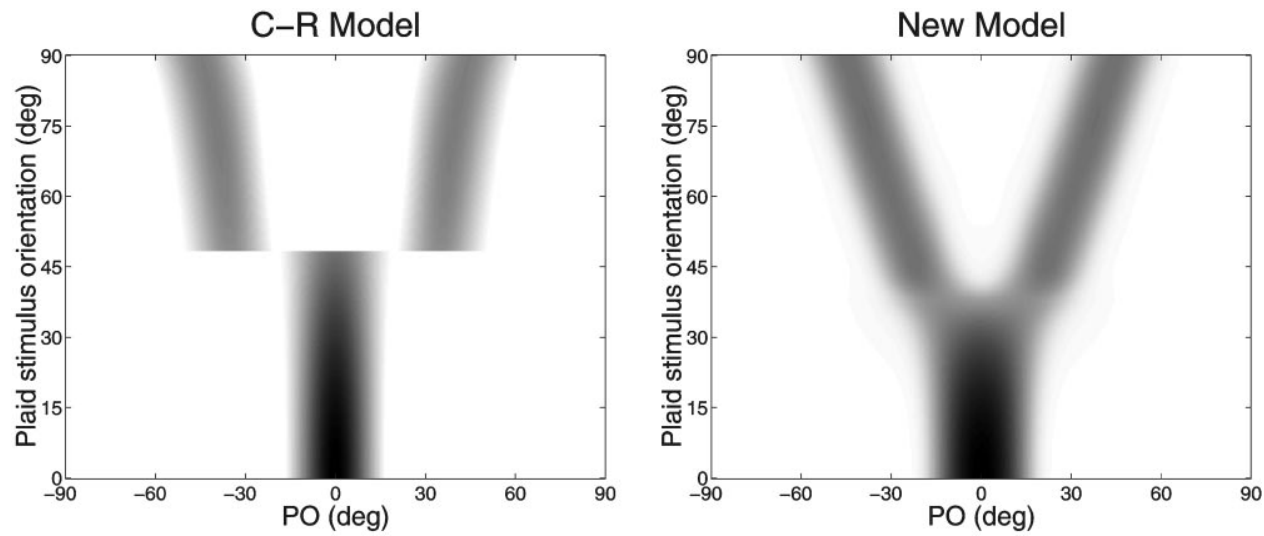

Fig. 3. Summary of responses to all two-orientation stimuli for both models. The model striate units are arranged according to their preferred orientation (PO). Each horizontal slice shows the unit activities to a particular two-orientation stimulus. The vertical axis represents the difference between the two orientations in the stimulus, growing from $0^{\circ}$ to $90^{\circ}$ (maximally orthogonal) separation. Note that the C-R model response is discontinuous near $45^{\circ}$, while our model contains a smooth transition.

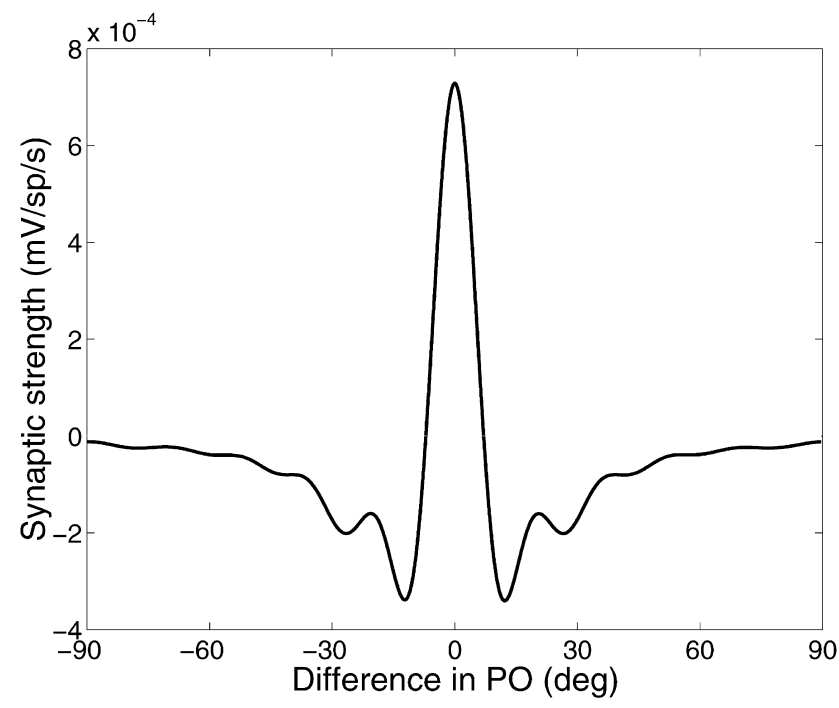

Fig. 4. The optimized recurrent connection strengths between pairs of model cells in our model are plotted as a function of the difference in their preferred orientations (POs). Recurrent input is computed by convolving this weight profile with the current firing rates. The weights' center-surround profile sharpens the weak orientation bias present in LGN input without destroying information about multiple orientations.

Our model makes a number of new predictions concerning responses of these cells to stimuli containing multiple orientations: (1) a range of stable activity patterns will be produced for stimuli containing two orientations with differing angular spread; (2) inputs containing three orientations separated by $60^{\circ}$ will produce trimodal 
activity patterns; and (3) noisy stimuli containing single orientations will only rarely give rise to spurious bimodal response profiles.

Each of these points differ from predictions derived from the Carandini-Ringach model. They also diverge from other models of orientation selectivity, such as that proposed by Pouget et al. [3]. This model forms stable activity patterns in the population such that simple decoding techniques, such as the population vector method, closely approximate maximum likelihood estimation, which is the optimal decoding technique. This model, however, responds to pure noise stimuli as if a single orientation is present, and cannot encode multiple orientations simultaneously.

Finally, we are currently exploring the consequences of this information preservation on processing downstream from V1. It is known that V2 cells respond to illusory contours and figure-ground information, and we hypothesize that preserving information about multiple orientations within individual V1 populations plays an important role in V2 responses.

\section{Acknowledgements}

This work was funded by ONR Young Investigator Award N00014-98-1-0509 to RZ. We thank Peter Dayan and Alexandre Pouget for many useful discussions of statistical population coding.

\section{References}

[1] M. Carandini, D.L. Ringach, Predictions of a recurrent model of orientation selectivity, Vision Res. 37 (21) (1997) 3061-3071.

[2] G.C. DeAngelis, J.G. Robson, I. Ohzawa, R.D. Freeman, Organization of suppression in receptive fields of neurons in cat visual cortex, J. Neurophysiol. 68 (1) (1992) 144-163.

[3] A. Pouget, K. Zhang, S. Deneve, P.E. Latham, Statistically efficient estimation using population codes, Neural Comput. 10 (2) (1998) 373-401.

[4] D.C. Somers, S.B. Nelson, R.J. Douglas, An emergent model of orientation selectivity in cat visual cortical simple cells, J Neurosci. 15 (1995) 6700-6719.

[5] H. Sompolinsky, R. Shapley, New perspectives on the mechanisms for orientation selectivity, Curr. Opin. Neurobiol. 7 (1997) 514-522.

[6] S. Treue, K. Hol, H.-J. Rauber, Seeing multiple directions of motion: physiology and psychophysics. Nature Neurosci. 3 (3) (2000) 270-276.

[7] G.H. Recanzone, R.H. Wurtz, U. Schwarz, Responses of MT and MST neurons to one and two moving objects in the receptive field, J. Neurophysiol. 78 (6) (1997) 2904-2915.

[8] R.S. Zemel, P. Dayan, A. Pouget, Probabilistic interpretation of population codes, Neural Comput. 10 (1998) 403-430.

[9] K. Zhang, Representation of spatial orientation by the intrinsic dynamics of the head-direction cell ensemble: a theory, J. Neurosci. 16 (1996) 2112-2126. 

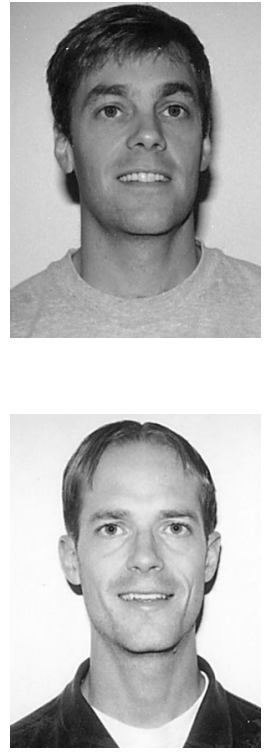

Richard Zemel is currently an Assistant Professor in the Departments of Psychology and Computer Science at the University of Arizona. He did four years of postdoctoral work at the Salk Institute and at Carnegie Mellon University before moving to Arizona. He obtained his undergraduate education at Harvard University, and his Ph.D. in Computer Science from the University of Toronto. His research interests include: machine learning, visual perception, and neural coding.

Jonathan Pillow was born in Tucson, Arizona to William and Deborah Pillow, and grew up in Phoenix, AZ, the elder of two boys. He graduated summa cum laude from the University of Arizona in 1997 with a BA in Mathematics and Philosophy. He subsequently studied north-African Literature in Morocco during a one-year tenure as a US Fulbright Scholar. He is currently a Ph.D. student at New York University in the Center for Neural Science. His research interests include information processing in the visual pathway, modelling of primary visual cortex, illusory contour perception, neural population coding, and analysis of timing and variability in neural spike trains. 